\title{
Use of nuclear data for space and aeronautic designs
}

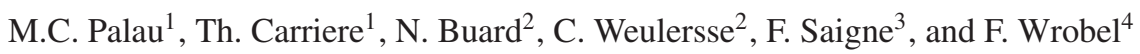 \\ 1 Astrium ST, 78133 Les Mureaux, France \\ 2 EADS IN, 92000 Suresnes, France \\ 3 CEM2, 34000 Montpellier, France \\ ${ }^{4}$ LPES, Nice University, France
}

\begin{abstract}
Until recently, the effects of radiation environment on on-board electronics on launchers and aircraft had not been seriously taken into account. The situation has changed. And one of the most significant effects observed on on-board electronics is what we call Single Event Upset (SEU). This talk explains how the combination of electrical sensitivity of components and nuclear physics is important in the calculation of SEU rates, and emphasizes the aspects of nuclear physics useful to give the probability for a dangerous event to occur. Some circumvention methods will be rapidly identified.
\end{abstract}

\section{Introduction}

Until now, the effects of radiation environment on-board electronics on launchers and aircraft had not been taken into account. But the situation has changed. For example, ARIANE 5 trajectories involve both altitudes of up to $36,000 \mathrm{~km}$ and regions densely populated with trapped protons, and integrated components being used are now manufactured on sub-micron technologies.

For aircraft, as well, altitudes are higher than by the past and electronic devices are playing an ever more significant role, involving increasingly sophisticated components.

After a description of the natural radiation environment, we would like to show how it can affect the behaviour of onboard electronics on launchers and on aircraft.

\section{Radiations environments}

Nuclear physics will deal closely with the definition of radiations environments as well for launcher as for civilian aircraft. The types of radiations contributing to both environments are not yet well-known and a lot of upstream research is still done as well to understand the Sun behaviour as to define the origin and path of galactic radiations and van Allen belts.

\subsection{Radiations environments associated with launch vehicles}

So far, radiation environments were of small interest to ARIANE series. But this situation has changed with ARIANE 5 [1]. On Solar Synchronous Orbit (SSO) trajectories, used for terrestrial observation satellite launching, ARIANE 5 passes through the polar horns and is affected by heavy ions produced by cosmic radiation [2]. On Geostationary Transfer Orbit (GTO) trajectories, the launch vehicle is affected by the trapped protons of the Van Allen belts. Figure 1 shows the natural radiation environment encountered by the launcher.

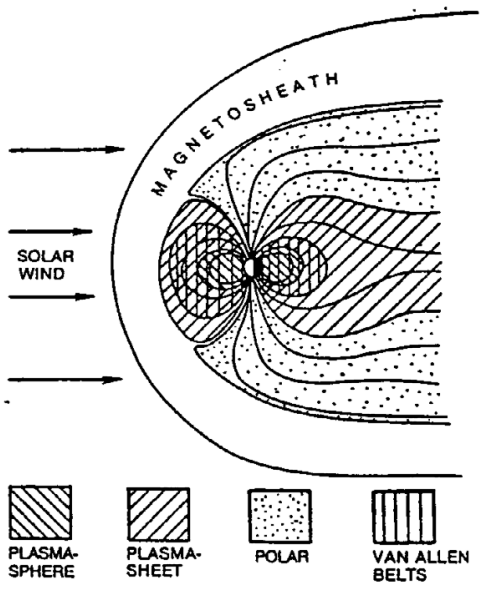

Fig. 1. Cross section of the Earth magnetic field.

As shown in figure 2, for a reference GTO mission, proton fluency to which ARIANE 5 is exposed is about two orders of magnitude greater than that associated with an ARIANE 4 flight. Furthermore, ARIANE 5 uses sub-micron component technologies whose radiation responses must be suitably tested; ARIANE 4 items of equipment were more conservative.

The type of radiation environment affecting ARIANE 5 depends on the launchers mission. In all cases, total dose remains negligible ( $50 \mathrm{rad}$ for a $4 \mathrm{~mm}$ aluminium shield) in the worst case scenario.

So, the four components to be considered are:

- protons trapped in radiation belts,

- solar protons (coronal mass ejection),

- heavy ions emitted by solar flares, and

- cosmic rays.

The four above mentioned components do not usually occur simultaneously: allowance for them in any given mission depends on what is being required of the launcher. At that time, we can identify five possible types of trajectories:

- reference GTO (Geostationary Transfert Orbit), 


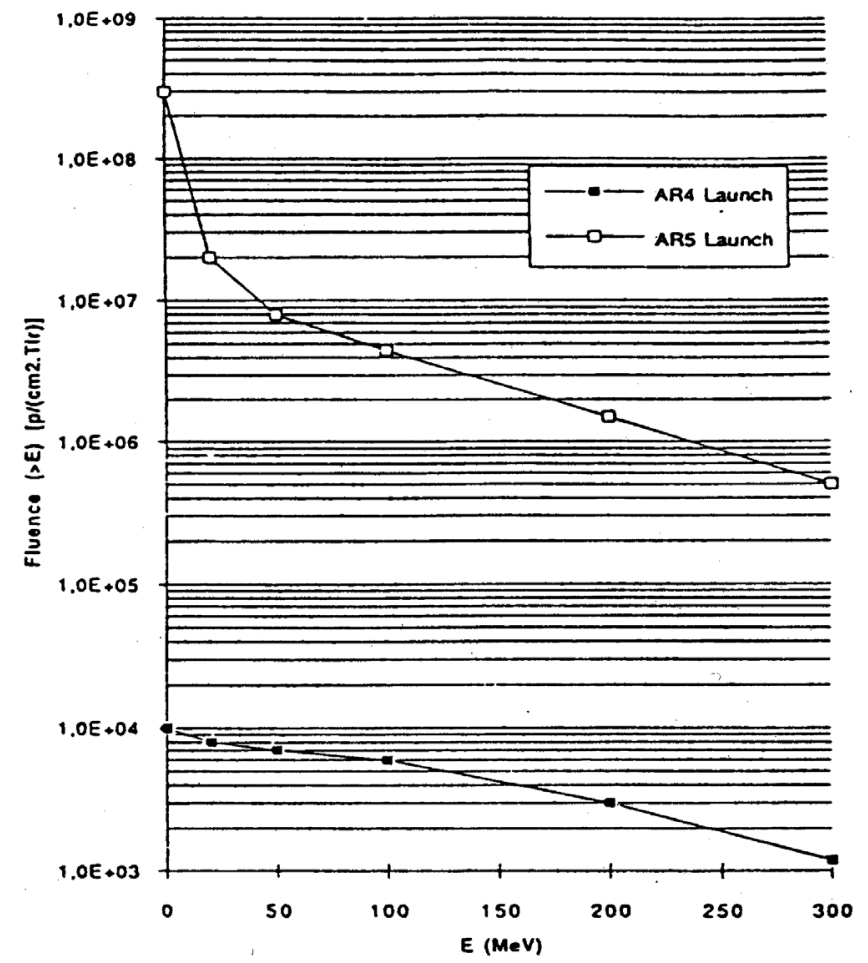

Fig. 2. Comparison between ARIANE 4 and ARIANE 5 protons environments.

- reference SSO (Solar Synchronous Orbit),

- XMM,

- Celestri,

- GTO +.

We only consider the two first missions.

The main characteristics of these trajectories are described hereafter.

\section{Reference GTO}

This type of trajectory applies to geostationary satellites used for telecommunications business. The satellite injection point is reached at an altitude of $1600 \mathrm{~km}$, after a $1600 \mathrm{~s}$ (about $30 \mathrm{mn}$ ) flight. In this type of trajectory, the launch vehicle is exposed primarily to protons trapped in the van Allen belt.

\section{Reference SSO}

This launch trajectory is used for helio synchronous satellites intended for Earth observation. The satellite injection point is located at an altitude of $830 \mathrm{~km}$ and is reached after a $2100 \mathrm{~s}$ ( $35 \mathrm{mn}$ ) flight. In this type of trajectory, the launcher passes through the polar horns. The source of constraints associated with this type of trajectory (i.e., protons, solar ions and cosmic rays) is its high latitude path (where magnetosphere screen effect is low).

As far as SSO missions are concerned, the calculation of the event factors likely to occur on the trajectory had been made. It appears that the measurements implemented to overcome the problems caused by protons are sufficient to guarantee the mission's success when it takes place under nominal solar weather conditions.
If a solar flare occurs, the problem changes because the instantaneous particle fluxes become highly penalizing, as shown on table 1.

A few items of basic information will help to understand this phenomenon:

- solar flare are more probable when solar activity is maximum,

- one flare out of 1000 is a particle eruption (protons or heavy ions)

- the first particles arrive, at the earliest, $20 \mathrm{mn}$ after the beginning of the flare and, on average, 20 hours later,

- a flare can last several days.

In order not to oversize the nominal protection of the launcher, it has been chosen to turn towards the detection of solar flares and, where applicable, to delay the launch to ensure that the mission takes place under optimum conditions. In that context, a procedure has been defined in order to avoid a launch during a solar flare and to try to detect the occurrence of such an event. The procedure is summarized in [3].

\subsection{Radiations environments closed to the Earth}

As early as 1984 [4], certain authors were predicting that atmospheric neutrons would cause Single Event Upsets in electronic devices. However, it was only in 1992 that the first in-flight upset was observed. Since then, several hundred upsets have been recorded by SRAMs during as many flights (of civilian and military aircraft) corresponding to several thousands of hours of flight time.

It has been demonstrated that these upsets are caused by neutrons. Nuclear interaction with a silicon nucleus causes emission of recoiling or spallation nuclei, i.e., an indirect heavy ion effect.

Atmospheric protons play a minor role and the impact of heavy ions is heavily mitigated by earth atmosphere.

Upsets registered by computer memories are causing more and more concern in avionics industry. In this field, as elsewhere, electronic devices are playing an ever more significant role, involving increasingly sophisticated components. It is likely that, in near future, direct proton-induced events will appear in operating in space.

Cosmic rays cover a large spectrum of energies (the established record exceeds $10^{14} \mathrm{MeV}$, but, in this case, flux is less than one particle per $\mathrm{km}^{2}$ per century). In most cases, the energies transported by these particles range from $100 \mathrm{MeV}$ to $10 \mathrm{GeV}$ with a peak in the vicinity of $500 \mathrm{MeV}$. Some of these "cosmic" particles collide with the nuclei of atoms making up the species present in the Earth's atmosphere. Nuclear reactions then produce "secondary cosmic rays" and elementary particles such as protons, muons, pions, electrons and gamma rays. Incident rays (or the secondary particles associated there with) in turn initiate what are called "air showers". The analysis of particle flux neutrons, protons, electrons, $\gamma$ - and X-rays) as a function of altitude (see fig. 3) shows a predominance of neutrons at aircraft altitudes. On the ground, the concentration of muons (deriving from pion decay) is higher than that of neutrons. 


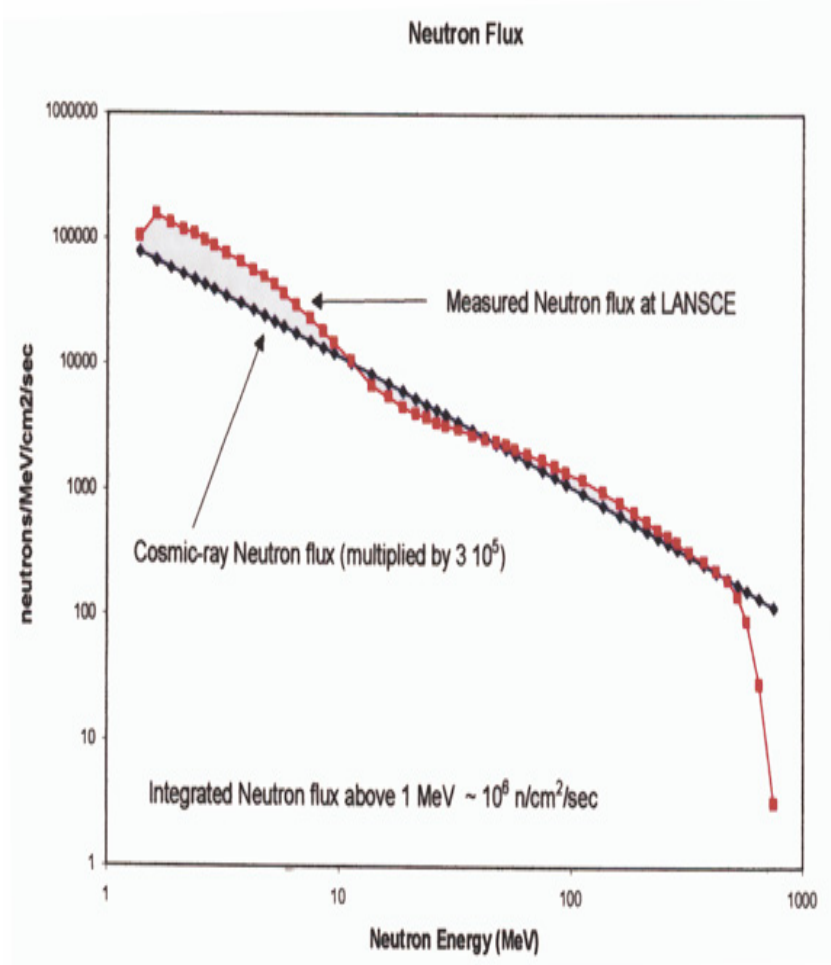

Fig. 3. Neutron energy spectrum.

\section{Effects of environment particles on microelectronics}

\subsection{Introduction}

Single Event Upsets (SEU) and Multiple Bit Upset (MBU) induced by primary ion or secondary ions have been clearly identified as a critical failure in integrated devices. MBU has been observed since 1990s and increase exponentially when gate length decreases [5,6]. Their understanding and impact in integrated components are still a challenge. The probability that two adjacent bits flip as a result of two separate neutron interaction is negligible. MBU are induced by secondary ions created by one neutron interaction. Neutrons do not have direct ionizing power and proton have a Linear Energy Transfer (LET) insufficient to induce upset. However, they can indirectly induce single events by creating secondary ions (recoils and fragments) due to a nuclear reaction with a nucleus of the component. And we find here how nuclear physics deals with particles components interaction.

The calculations of soft error rates (SER) are still a challenge to obtain the technology sensitivity and to extrapolate the trends for integrated devices. Methods have been proposed, using combined nuclear codes and device simulations or semiempirical coupling of nuclear physics and experimental data [7-11]. Since 1998, a neutron-silicon nuclear database was established and updated [10] and in 2001, Hubert et al. [11] proposed a new approach based on nuclear database coupling with occurrence criteria deduced from a Detailed Analysis of Secondary Ion Effect (DASIE).

\subsection{Physical mechanisms involved in the transient creation}

In order to estimate the soft error rate due to neutrons and protons in CMOS logic, we consider at first the nuclear reactions and the secondary ions produced in the device. Then, an adequate model allows calculating the transient current pulse in the sensitive node. Finally, the transient current pulse is introduced in a circuit simulation.

\subsection{1 $\mathrm{n} / \mathrm{p}$ nuclear reaction}

In silicon, secondary particles created by nuclear interactions can range from $\mathrm{H}$ to $\mathrm{P}$, including several isotopes. Nuclear reactions can appear anywhere inside the interaction volume $V_{\text {int }}$. Large variations in the properties of the secondary ions created by nuclear reactions of protons and neutrons (nucleons) with silicon may appear $[10,11]$. In fact, several secondary ions can be simultaneously generated by a specific nuclear reaction. The number of secondary ions produced depends (but not only) on the nucleon energy: for example, $63 \mathrm{MeV}$ and $14 \mathrm{MeV}$ neutrons can induce respectively 1 to 4 and 1 to 2 secondary ions. Nuclear databases have been created for proton-silicon and neutron-silicon interactions. These databases have been built by using a Monte Carlo code, called MC-RED1.2 [13], in the 1-200 MeV energy range. This code treats non-elastic reactions with the exciton model (pre-equilibrium step) and the Hauser-Feschbach formulation (equilibrium step). Elastic reactions are treated with the ECIS code thanks to optical models from RIPL (Recommended Input Parameters Library). Nuclear databases are made with:

- the detail of the nuclear reactions, that is, the atomic and mass number of the secondary ions, their energies and directions.

- the nuclear cross section values relative to the elastic $\left(\sigma_{\text {elastic }}\right)$ and nonelastic $\left(\sigma_{\text {nonelastic }}\right)$ reaction types.

As mentioned before, large variations in the secondary ion properties generated by nuclear reactions may appear. For example, for the same energy, a heavy secondary ion, which has a high LET and a short range, is more likely to cause a transient pulse than a light one, whose LET is smaller and range greater. But, to observe a single event effect, the heavy ion requires to be generated close to the sensitive region while the light ion can be generated farther. Silicon recoils provoke transients close to their generation point, while light ions, particularly $\mathrm{He}$ and $\mathrm{H}$, can be created several tens or hundreds of micrometers away from the generation point, and induce transient.

\subsubsection{Physical mechanisms induced by secondary ions}

The secondary ions created by a nuclear reaction induce electron-hole pairs along their track by Coulombian interactions with electrons [14]. Ambipolar diffusion then collection of the created carriers can induce transient current pulses in the external circuit. Figure 4 presents a synthesis of the physical mechanisms inducing transient pulse. 


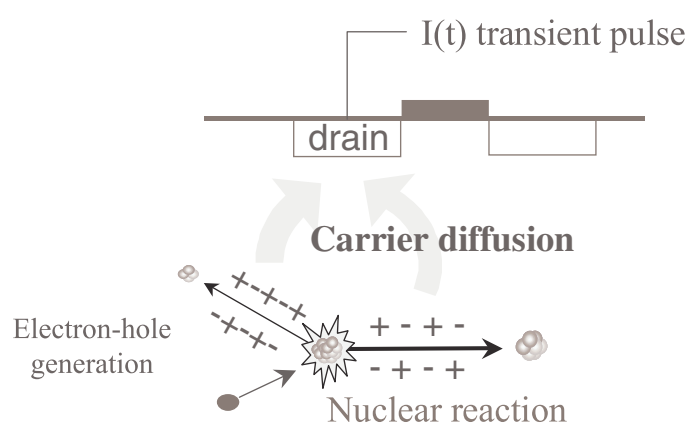

Fig. 4. Physical mechanisms induced transient pulse.

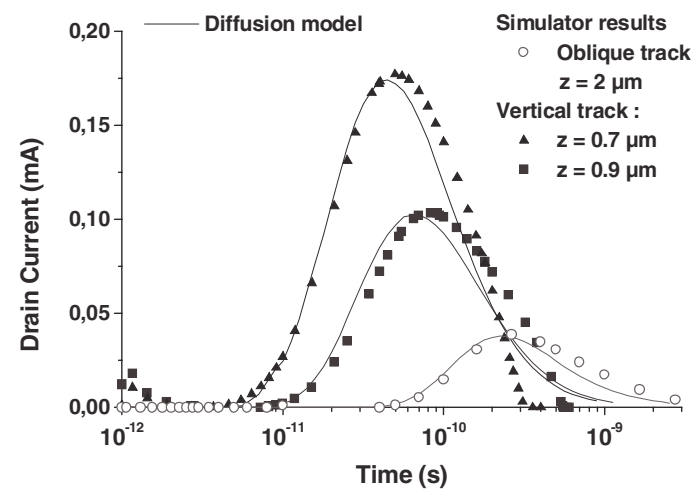

Fig. 5. Currents obtained by device simulation and diffusion model [15].

In practice, several 3D full-cell device simulations were performed in previous investigations with various track configurations $[15,16]$. These 3D device simulations show that transient current pulse is due to the charge collection phenomenon at the junction from tracks arriving after ambipolar diffusion. The drain currents induced by secondary ions and obtained by 3D device simulation were compared towards a diffusion-collection model based on ambipolar diffusion laws. Good agreement was obtained for the neighbouring tracks. As a conclusion, diffusion phenomenon was clearly identified as the main mechanism in transient pulse generation.

To illustrate the relevance of the diffusion model, figure 5 presents the drain-current obtained from device simulation compared with the diffusion model [15].

The current pulse can be delayed with respect to the impact time. This delay depends on the distance between the track and the collecting area, and is basically due to the time needed for the excess carriers to reach the collecting areas. Obviously, there is no delay when an ion shunts the electrodes and the charge collection can begin immediately, resulting in a very short transient.

As a second conclusion, a simple diffusion-collection model allows to calculate the transient current pulse collected by the sensitive area, thus avoiding very expensive long $3 \mathrm{D}$ device simulations.

\subsubsection{Transient pulse calculation}

When the ion track is sufficiently far from the space charge zone of the junction, the carriers generated in tracks mainly

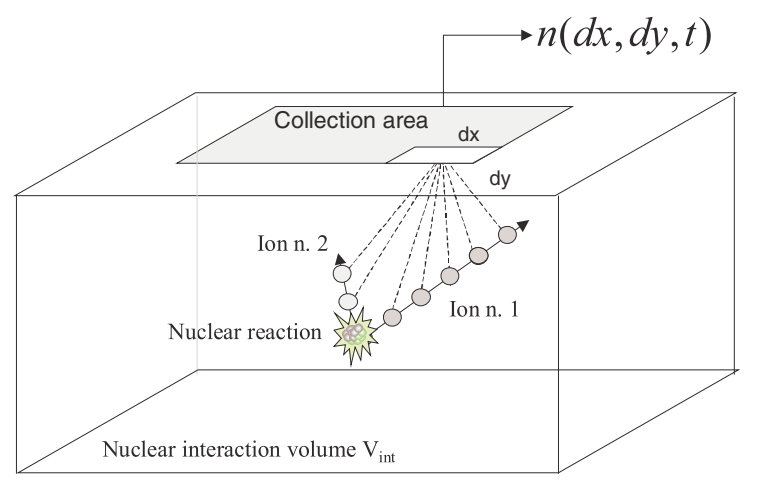

Fig. 6. Current pulse calculation method.

move by diffusion. We consider that recombination is negligible and that the space charge zone of the sensitive zone collects all the carriers that are crossing it. Let us consider some simple geometry cases for which the pure diffusion equation can be solved analytically:

$$
\frac{\partial n}{\partial t}=D \cdot \Delta n
$$

where $n$ is the density of electrons in the conduction band, obviously equal to the hole density $p$ and $D$ the ambipolar diffusion constant. The simplest case is the one of spherical diffusion, when electron-hole pairs are deposited in a very small volume and are diffusing in a spherical way.

It is possible to consider the ion tracks as a lot of punctual carrier density $n_{D}^{i}$. The location of each punctual carrier density is characterized by a distance $\rho_{i}$ from the collected area. Each punctual carrier density is governed by a spherical diffusion law allowing to calculate the carrier density arriving in the collected area. This carrier density is given by:

$$
n(d x, d y, t)=\sum_{i} n_{D}^{i} \cdot \frac{e^{-\frac{\rho_{i}^{2}}{4 \cdot D . t}}}{(4 \cdot \pi \cdot D \cdot t)^{3 / 2}} .
$$

The current collected by the collected area is calculated with the following formula:

$$
I(t)=\iint_{\text {drain }} q \cdot n(d x, d y, t) \cdot v \cdot d x d y
$$

where $q$ is the electrical charge $\left(1.6 \cdot 10^{-19} \mathrm{C}\right), d x \cdot d y$ the elementary surface and $v$ the collection velocity. Figure 6 presents the $I(t)$ transient calculation method.

\section{Simplified MC-DASIE description}

\subsection{Presentation of S-MC DASIE}

We consider the ion track like as a serie of punctual charges $Q_{D}^{i}$ whose the location is characterized by a distance $\rho_{\mathrm{i}}$ to the drain then and by collection efficiency value $E f f\left(\rho_{i}\right)$. The total collected charge is given by $\mathrm{Q}_{\text {coll }}=\sum_{i} Q_{D}^{i} \cdot \operatorname{Eff}\left(\rho_{i}\right)$.

Figure 7 presents the collected charge calculation method used in S-MC DASIE. This method allows calculating the 


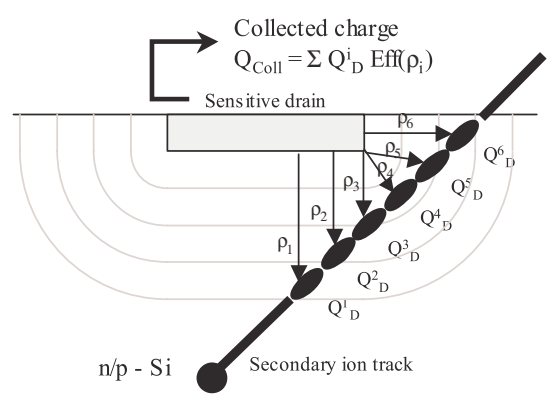

Fig. 7. Collected charge calculation method developed in S-MC DASIE.

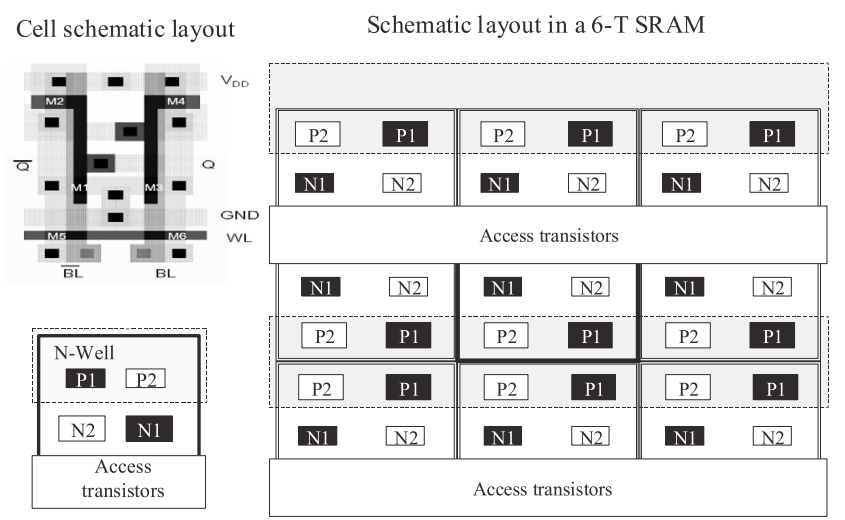

Fig. 8. One cell layout (left) and 6T SRAM schematic layout (right) in S-MC DASIE ("All zeros" data pattern).

collected charge for one drain. It is possible to extend the calculation to numerous drains. Figure 8 schematizes a 6T RAM cell and memory: access transistors area and four locations corresponding to potential sensitive drains.

Either $(\mathrm{N} 1, \mathrm{P} 1)$ or $(\mathrm{N} 2, \mathrm{P} 2)$ are the sensitive drains depending of the logic cell state. The calculation of collected charge by the off-state drains allows to determine, with an relevant charge criterion, the occurrence of events. S-MC DASIE allows to consider any data pattern. In our investigations, we will consider the "All 0" and "Checkerboard" data pattern.

Figure 9 presents a flow chart of S-MC DASIE code: depending on neutron/proton energies or spectrum, secondary ions are generated by Monte Carlo process thanks to nuclear database. The critical deposited charge $\mathrm{Q}_{\mathrm{CD}}$ is calculated for each cell and compared to upset criteria.

S-MC DASIE input parameters are limited. The geometry drain characteristics, i.e., the drain length $\mathrm{L}_{d}$ and drain locations are considered. It is necessary to define the critical charge $\mathrm{Q}_{\mathrm{N}}$ and the critical LET. Cell spacing is defined as an multiple of gate length ranging from 15-20 times. The whole structure simulated by S-MC DASIE contain up to $2 \mathrm{k} \times 2 \mathrm{k}$ cells and the generated depth is about $10.000 \mu \mathrm{m}$. In comparison to the classical MC-DASIE method, the S-MC DASIE calculation time is very fast, typically about 1 hour (PC).

\subsection{S-MC DASIE code validation}

S-MC DASIE results can be compared to experimental results. Figures 10 and 11 present a comparison between experimental

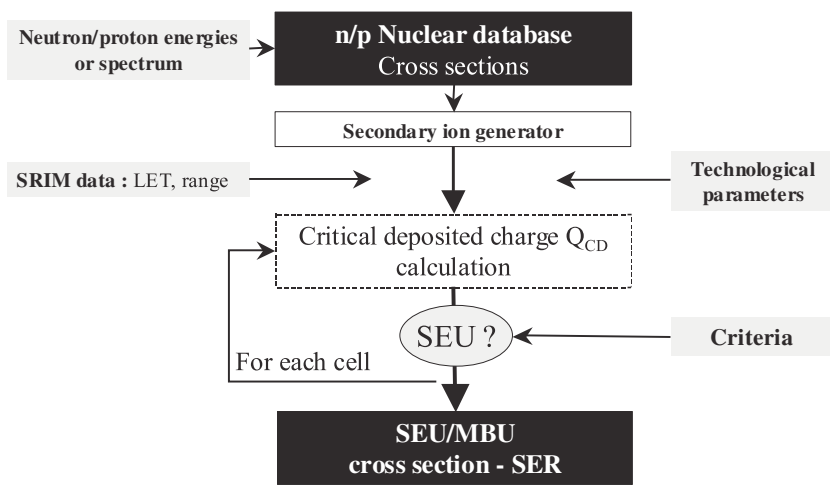

Fig. 9. Flowchart of S-MC DASIE.

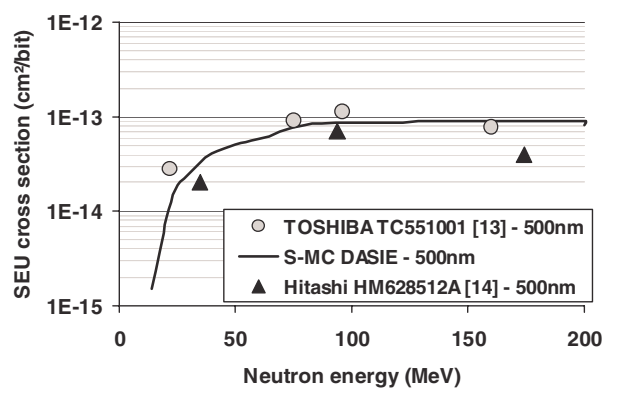

Fig. 10. Experimental and calculated SEU cross sections obtained for two $500 \mathrm{~nm}$ bulk devices (TOSHIBA TC551001 [17] and Hitachi HM628512A [18]).

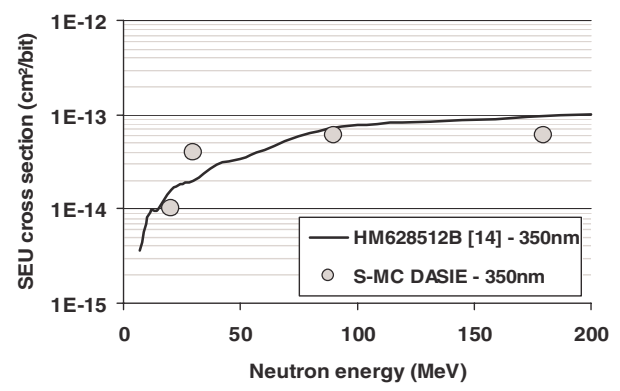

Fig. 11. Experimental and calculated SEU cross sections obtained for $350 \mathrm{~nm}$ bulk device (Hitachi HM628512B [18]).

and calculated SEU cross section obtained for some $500 \mathrm{~nm}$ and $350 \mathrm{~nm}$ SRAM. S-MC DASIE parameters used are respectively $\left(\mathrm{Q}_{\mathrm{N}}=30 \mathrm{fC} ; \mathrm{L}_{\mathrm{d}}=1.5 \mu \mathrm{m}\right)$ for $500 \mathrm{~nm}$ and $\left(\mathrm{Q}_{\mathrm{N}}=12 \mathrm{fC}\right.$; $\mathrm{L}_{\mathrm{d}}=1 \mu \mathrm{m}$ ) for $350 \mathrm{~nm}$.

The drain area $S_{d}$ was been given by manufacturers. The critical LET is determined by heavy ions experiments and the critical charge $Q_{N}$ is chosen in agreement with node technology. A good agreement between calculation and experimental data has been obtained for several SRAM. 


\section{Circumvention and conclusions}

It has been demonstrated that nuclear data help a lot as well as when radiation environments must be precise as when radiations-components interaction must be understood.

A lot of work has to be done to go further in that two domains: we are confident to the Sun nuclear physicists and the Deep Space physicists to understand Space around the Earth and we need more databases (heavy ions- silicon interaction for example) to defined what could be the exact influence of material in the final SEU or MBU rate inside the component.

There is something in the domain nuclear physics can't help at all: circumvention implementation.

The work that has been done in ref. [1] gives the main requests that have been given during ARIANE 5 design phase: we can summarize them in a few sentences:

- Choice of less sensitive components,

- Introduction of EDAC on mass memory,

- Use of a redundancy mode,

- Software protection.

Consequent studies have been done in another specific domain which shows that System Circumvention technology has a large future in front of it.

Science is running and industry needs more and more collaboration with high level scientific laboratories to explore al that specific domains.

\section{References}

1. M.C. Calvet et al., Acknowledgement of the Natural Radiation Environment upon the ARIANE5 Launcher, RADECS 95.

2. J. Bourrieau, Irradiations, Doses Cumulées, Ions Lourds, Cours de Technologie Spatiale, CNES, March 1988.

3. M.C. Calvet, J. Bourrrieau, P. Lantos, E.G. Stassinopoulos, An industrial method to predict major solar flares for better protection of human beings in space, 1997 Man in Space conference.
4. Silberberg et al., Neutron generated Single Event Upset in the atmosphere, IEEE-TNS 31-6.

5. J. Maiz et al., Characterisation of Multi-bit Soft Error Events in Advanced SRAMs, IEDM, 2003.

6. T. Merelle et al., Monte-Carlo Simulations to quantify neutroninduced Multiple Bit Upsets in advanced SRAMs, RADECS, 2004.

7. E. Normand, Single Event Upset at Ground Level, IEEE Trans. Nucl. Sci., Vol. 43 (1996).

8. M.B. Chadwick et al., Use of New ENDF/B-VI Proton and Neutron Cross Sections for Single Event Upset Calculations, IEEE Trans. Nucl. Sci., Vol. 46, No. 6 (1999), p. 1386.

9. H.K. Tang et al., SEMM-2: A modeling System for Single Event Analysis, IEEE Trans. Nucl. Sci., Vol. 51, No. 6 (Dec. 2004).

10. F. Wrobel, Database of particles induced failure in electronic component in protons and neutrons environment, Thesis, 2002.

11. G. Hubert et al., Detailed analysis of secondary ions' effect for the calculation of neutron-induced SER in SRAMs, IEEE Trans. Nucl. Sci., Vol. 48, No. 6 (2001).

12. G. Hubert et al., Review of DASIE Family Code: Contribution to SEU/MBU Understanding, $11^{\text {th }}$ IEEE International On-Line Testing Symposium 2005, Saint-Raphaël, France.

13. F. Wrobel et al., Use of Nuclear Codes for Neutron-Induced Reactions in Microelectronics, $11^{\text {th }}$ IEEE International On-Line Testing Symposium 2005, Saint-Raphaël, France.

14. G. Hubert et al., Study of basic mechanisms induced by an ionizing particle on simple structures, IEEE Trans. Nucl. Sci., Vol. 47 (2000), pp. 519-526.

15. Ph. Roche et al., Determination on Key Parameters for SEU using Full Cell 3-D SRAM Simulations, IEEE Trans. Nucl. Sci., Vol. 46 (1999), pp. 1354-1362.

16. Palau et al., Device Simulation Study of the SEU Sensitivity of SRAMs to Internal Ion Tracks Generated by Nuclear Reactions, IEEE Trans. Nucl. Sci., Vol. 48 (2001).

17. K. Johansson et al., Energy-resolved neutron SEU measurements for 22 to $160 \mathrm{MeV}$, IEEE Trans. Nucl. Sci., Vol. 45, No. 6 (Dec. 1998).

18. C.S. Dyer et al., An experimental study of single-event effects induced in commercial SRAMs by neutrons and protons from thermal energies to $500 \mathrm{MeV}$, IEEE Trans. Nucl. Sci., Vol. 51 (Oct. 2004). 